\title{
Alkoholabhängigkeit \\ Reduktion der Trinkmenge als Strategie
}

\author{
Die Zahl spricht für sich: Für etwa neun Millionen Menschen besteht \\ aufgrund von Alkoholabhängigkeit oder schädlichem beziehungs- \\ weise riskantem Konsum ein Beratungs- oder Behandlungsbedarf.
}

\begin{abstract}
$V^{\infty}$ on diesen neun Millionen rechnet man 1,2 bis 1,6 Millionen definitiv zu den Alkoholkranken, so Professor Michael Soyka aus Meiringen/Schweiz beim Psychiatrie-Update in Mainz. Der eindeutigste Risikofaktor für Alkoholismus ist die Verwandtschaft ersten Grades mit einer Person, die ein Alkoholproblem hat.

Relevant ist die Komorbidität mit anderen psychischen Erkrankungen. So verdoppelt besonders in der Adoleszenz (14. bis 17. Lebensjahr) eine Suchterkrankung das Risiko einer depressiven Stö-
\end{abstract}

rung im jungen Erwachsenenalter. Umgekehrt erhöhen Angsterkrankungen das Alkoholismusrisiko um den Faktor zwei bis drei, bipolare Störungen sogar um das Sechsfache. Mit der Trinkmenge steigt auch das Risiko für Folgeschäden exponenzial an, so Soyka. Neben der Abstinenz ist daher die Reduktion der Trinkmenge ein Kriterium der Wirksamkeit für eine Rückfallprävention. Als wirksame psychotherapeutische Verfahren haben sich soziales Kompetenztraining, kognitive und verhaltensbasierte Therapien sowie paar- und familientherapeutisch orientierte Ansätze erwiesen. Damit lassen sich längerfristig Abstinenzraten von $40 \%$ bis maximal $50 \%$ erzielen. Das evidenzbasierte Arsenal der Medikamente, die nach einem Entzug die Rückfallrate reduzieren, ist mit Acamprosat und dem Opiatantagonisten Naltrexon sehr klein. Während Acamprosat nach CochraneDaten im Gegensatz zu Naltrexon die Rückfallrate in gewissem Ausmaß senkt, ist unter Naltrexon die Trinkmenge etwas stärker reduziert. Noch ausgeprägter ist dieser Effekt bei der demnächst verfügbaren neuen Substanz Nalmefen, die sowohl den My- als auch Kappa-Opiatrezeptor hemmt. Für Baclofen besteht nach Soykas Ansicht dagegen kaum Evidenz.

Andreas Häckel 